\title{
Oral lactoferrin influences psychological stress in humans: A single-dose administration crossover study
}

\author{
TOKIKO SHINJO $^{1}$, KEISHOKU SAKURABA ${ }^{1,2}$, ATSUKO NAKANIIDA ${ }^{2}$, \\ TOMOYO ISHIBASHI $^{2}$, MIKI KOBAYASHI ${ }^{2}$, YUYAAONO ${ }^{2}$ and YOSHIO SUZUKI ${ }^{1,2}$ \\ ${ }^{1}$ Graduate School of Health and Sports Science; \\ ${ }^{2}$ Faculty of Health and Sports Science, Juntendo University, Chiba 270-1695, Japan
}

Received December 28, 2017; Accepted March 5, 2018

DOI: $10.3892 / b r .2018 .1076$

\begin{abstract}
Lactoferrin is a secretory protein with various physiological functions. Bovine lactoferrin has been demonstrated to alleviate psychological stresses in rats, but this effect in humans has not yet been assessed. The present study aimed to investigate the changes in psychological stress markers following a calculation task, with either lactoferrin or a placebo orally administered prior to the task. A total of 16 healthy female college students visited Juntendo University, Inzai, Japan following an overnight fast. Subjects were quietly seated for $15 \mathrm{~min}$ to stabilize the respiratory rate at $0.25 \mathrm{~Hz}$ (one breath every $4 \mathrm{sec}$ ). Then, subjects provided saliva, ingested either lactoferrin (800 mg of lactoferrin + soy milk) or a placebo (soy milk), remained seated for another $15 \mathrm{~min}$ with respiration rate at $0.25 \mathrm{~Hz}$, and performed a calculation task. The task comprised two sets of 15-min calculations, with a 5-min interval between sets. Each calculation set consisted of various multiplications and divisions using pairs of three-digit numbers. Following the calculation task, saliva was collected again. Heart rate was also monitored to identify the frequency domain of heart-rate variability. The calculation task resulted in increased activity of salivary amylase, and decreased concentration of chromogranin A for both lactoferrin ( $\mathrm{P}=0.028$ and $\mathrm{P}<0.001$, respectively) and placebo $(\mathrm{P}=0.003$ and $\mathrm{P}<0.001$, respectively) treatments. The degrees of changes in these salivary markers were similar between the two treatments. Heart rate variability exhibited an increase in the high-frequency $(\mathrm{HF})$ component $(\mathrm{P}=0.022)$ and a decrease in low-frequency ( $\mathrm{LF}) / \mathrm{HF}$ and $\mathrm{LF} /(\mathrm{LF}+\mathrm{HF}$ ) ratios (both $\mathrm{P}<0.001)$ following the calculation task under the placebo condition, demonstrating an upregulation of parasympathetic and a downregulation of sympathetic nervous activities. These
\end{abstract}

Correspondence to: Dr Yoshio Suzuki, Graduate School of Health and Sports Science, Juntendo University, 1-1 Hiragagakuendai, Inzai, Chiba 270-1695, Japan

E-mail: yssuzuki@juntendo.ac.jp

Key words: lactoferrin, psychological stress, amylase, heart rate variability, chromogranin A, autonomic nervous system changes in parasympathetic (HF) and sympathetic (LF/HF) activities, however, were alleviated by lactoferrin compared with the placebo $(\mathrm{P}=0.007$ and $\mathrm{P}=0.026$, respectively). Collectively these results suggest that oral lactoferrin may mitigate psychological stress in humans.

\section{Introduction}

Lactoferrin is a secretory protein present in mammalian secretary fluids, and has roles in protecting the body from external pathologies (1-6). It is also involved in prevention of cancer (7) and anemia $(8,9)$.

Lactoferrin has been demonstrated to alleviate psychological stresses in rats: Intraperitoneal administration of bovine lactoferrin suppressed the behavioral symptoms of stress in fear-induced freezing and elevated plus-maze tests (10). However, research on lactoferrin as an aid to manage psychological stresses has been limited to animal studies. Therefore, it remains unclear whether lactoferrin is capable of alleviating psychological stresses in humans. The present study, therefore, examined the effect of bovine lactoferrin ingestion on psychological stresses in human subjects. The psychological stresses were induced by facing subjects with calculation problems.

In the present study, the levels of psychological stresses were evaluated by means of salivary stress markers, namely the level of amylase activity $(11,12)$ and the concentration of chromogranin A (13-15). The levels of these stress markers have been reported to be increased in response to physical stresses, notably at an intensity greater than $70 \% \mathrm{VO}_{2} \max$ in healthy young individuals (16). Psychological stresses may also trigger their production. For instance, it has been reported that amylase activity was increased in subjects undergoing a written examination (17) and the Trier Social Stress Test (TSST) $(11,18)$. Similarly, chromogranin A concentration was increased in subjects receiving venipuncture (14), giving an oral presentation, or driving a car (19).

Autonomic nervous activities may also be assessed by frequency domain analysis of the R-R intervals of the heartbeat (20). This analysis provides the powers of low-frequency (LF; 0.04-0.15 Hz) and high-frequency (HF; 0.15-0.4 Hz) components (20). The power of the frequency domain to the LF component indicates the activities of the sympathetic and parasympathetic nervous systems, whereas that to the HF 
component signifies parasympathetic activity. Accordingly, increased $\mathrm{LF} / \mathrm{HF}$ and $\mathrm{LF} /(\mathrm{LF}+\mathrm{HF})$ ratios are indicative of increased sympathetic activity (21).

To the best of our knowledge, the present study is the first to investigate the potential of lactoferrin for managing psychological stresses incurred during human activity, using the aforementioned heart rate variables and salivary stress markers.

\section{Materials and methods}

Subjects. A total of 18 healthy female college students were recruited for the current study at Juntendo University, Inzai, Japan between 1 and 10 September, 2015. Their salivary amylase activities were confirmed to be elevated by the calculation task used in the trial (described below) prior to the study. All subjects read the guidelines detailing the purpose, methods and ethical considerations (including possible adverse effects) associated with the study, and provided written informed consent prior to participation. Among the participants, 2 subjects dropped out of the study for personal reasons. The remaining 16 subjects completed the study. Their age, height, body mass and body mass index were respectively $19.0 \pm 0.6$ years, $158.6 \pm 5.0 \mathrm{~cm}, 54.5 \pm 4.3 \mathrm{~kg}$ and $21.7 \pm 1.7$ $\mathrm{kg} / \mathrm{m}^{2}$ (mean \pm standard deviation). The research protocol was reviewed and approved by the research ethics committee of the Graduate School of Health and Sports Science, Juntendo University (Inzai, Chiba, Japan; approval no. 27-54). The study was registered in the UMIN Clinical Trials Registry (UMIN ID: UMIN000031319).

Study design. The present study employed a single-dose administration, double-blinded placebo-controlled trial in a crossover fashion. Subjects visited the institution on two occasions that were separated by 4 days. On the day prior to each experiment, subjects refrained from vigorous exercise and finished dinner by 9:00 p.m., and fasted on water until the experiment.

On the experiment day (Fig. 1), subjects visited the institution at 8:30 a.m., wore a heart-rate monitor (RS 800CX; Polar Japan, Tokyo, Japan) and rinsed their mouths with water. Each subject was quietly seated for $15 \mathrm{~min}$, while stabilizing the respiratory rate to breathe once every $4 \mathrm{sec}$ (equivalent to $0.25 \mathrm{~Hz}$ ) using metronome software (Art Metronome version 1.8; obtained from http://www. asahi-net.or.jp/ hb9t-ktd/music/Japan/Soft/metronome.html; accessed July, 2015). Heart rate was recorded during this rest period [Pre-condition (PRE); Fig. 1]. Saliva ( 2 ml) was then collected (PRE) using Salivette ${ }^{\circledR}$ (Sarstedt AG and Co., Nümbrecht, Germany), followed by an oral ingestion of either bovine lactoferrin or a placebo (details provided below). Following the ingestion, subjects remained seated for another 15 min while maintaining the respiratory rate at $0.25 \mathrm{~Hz}$, and began a calculation task. The calculation task comprised of two sets of 15-min calculations with a 5-min interval between sets. In each set of calculations, subjects attempted to solve 60 questions involving multiplications and divisions using pairs of three-digit numbers. Heart rate was recorded again during the second calculation set (Calc 2). Upon completion of the calculation task, subjects rinsed their mouths with water and provided saliva $(\sim 2 \mathrm{ml})$ post-condition (POST) using Salivette ${ }^{\circledR}$.
Lactoferrin and placebo. A total of 8 lactoferrin tablets (Morinaga Lactoferrin Original; Morinaga Milk Industry, Co., Ltd., Tokyo, Japan), containing $100 \mathrm{mg}$ of lactoferrin per tablet, were grained and dissolved in $150 \mathrm{ml}$ of soy beverage 'soymilk drink malt coffee calorie 50\% off' (Marusanai, Co., Ltd., Aichi, Japan). The same amount of the soymilk was used as a placebo. The appearance and the taste of the two test drinks were indistinguishable as judged by the examiners.

Salivary analyses. Salivary amylase activity (U/ml) and chromogranin A (pmol/ml) were analyzed with CicaLiquid-N AMY (Kanto Chemical, Co., Inc., Tokyo, Japan) and YK 070 Human Chromogranin A EIA (Yanaihara Institute, Inc., Shizuoka, Japan) kits, respectively, according to the manufacturer's protocols.

Frequency analysis of heart rate variability. Heart rate variability was analyzed using MemCalc version 2.0 software (GMS Co., Ltd., Tokyo, Japan) from R-R intervals obtained from the heart rate monitor, with segment length of $30 \mathrm{sec}$ and segment interval of $2 \mathrm{sec}$. The average values of power spectral density in LF $(0.04-0.15 \mathrm{~Hz})$ and HF $(0.15-0.4 \mathrm{~Hz})$ components, and the ratios $\mathrm{LF} / \mathrm{HF}$ and $\mathrm{LF} /(\mathrm{LF}+\mathrm{HF})$ were calculated.

Statistical analysis. Salivary amylase activity and chromogranin A concentration as well as variables of heart-rate variability were analyzed using a generalized linear model with the following predictor variables: i) Subject variable was 'subject ID'; and ii) within-subject variables were 'condition' (lactoferrin or placebo), 'experiment day' (date) and 'measurement' (PRE, POST or PRE/Calc 2). Estimated marginal means of 'condition x measurement' were compared. All interactions were confirmed and the model with the smallest quasi-likelihood under independence model criterion was adopted.

Changes in salivary amylase activity and chromogranin A concentration (POST-PRE) and the ratio of heart-rate variables (Calc 2/PRE) were also assessed in the same manner used to compare the estimated marginal mean of 'condition'.

All statistical analyses were performed using SPSS version 23 software (IBM Corp., Armonk, NY, USA). P<0.05 was considered to indicate a statistically significant difference.

\section{Results}

Salivary stress markers. For both placebo and lactoferrin treatment conditions, following the calculation task (POST), amylase activity was elevated $(\mathrm{P}=0.003$ and $\mathrm{P}=0.028$, respectively; Fig. 2A), while chromogranin A concentration was reduced (both $\mathrm{P}<0.001$; Fig. $2 \mathrm{~B}$ ).

It appeared that the magnitude of change in amylase activity following the calculation task was smaller $(\mathrm{P}=0.33$; Fig. 2C; ), and of the change in chromogranin A concentration greater $(\mathrm{P}=0.093$; Fig. 2D) for the lactoferrin treatment compared with the placebo; however, these differences were not statistically significant.

Heart-rate variability. With the placebo, the calculation task had no effect on the LF component (Fig. 3A), whereas it increased the HF component ( $\mathrm{P}=0.022$; Fig. 3B). Consequently, 


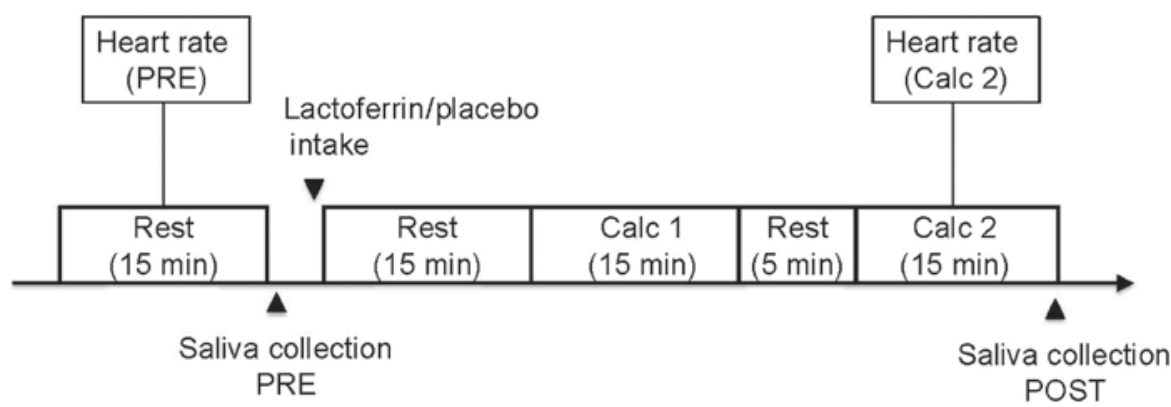

Figure 1. Summary of the experimental day procedure. PRE, pre-condition; POST, post-condition; Calc, calculation task.
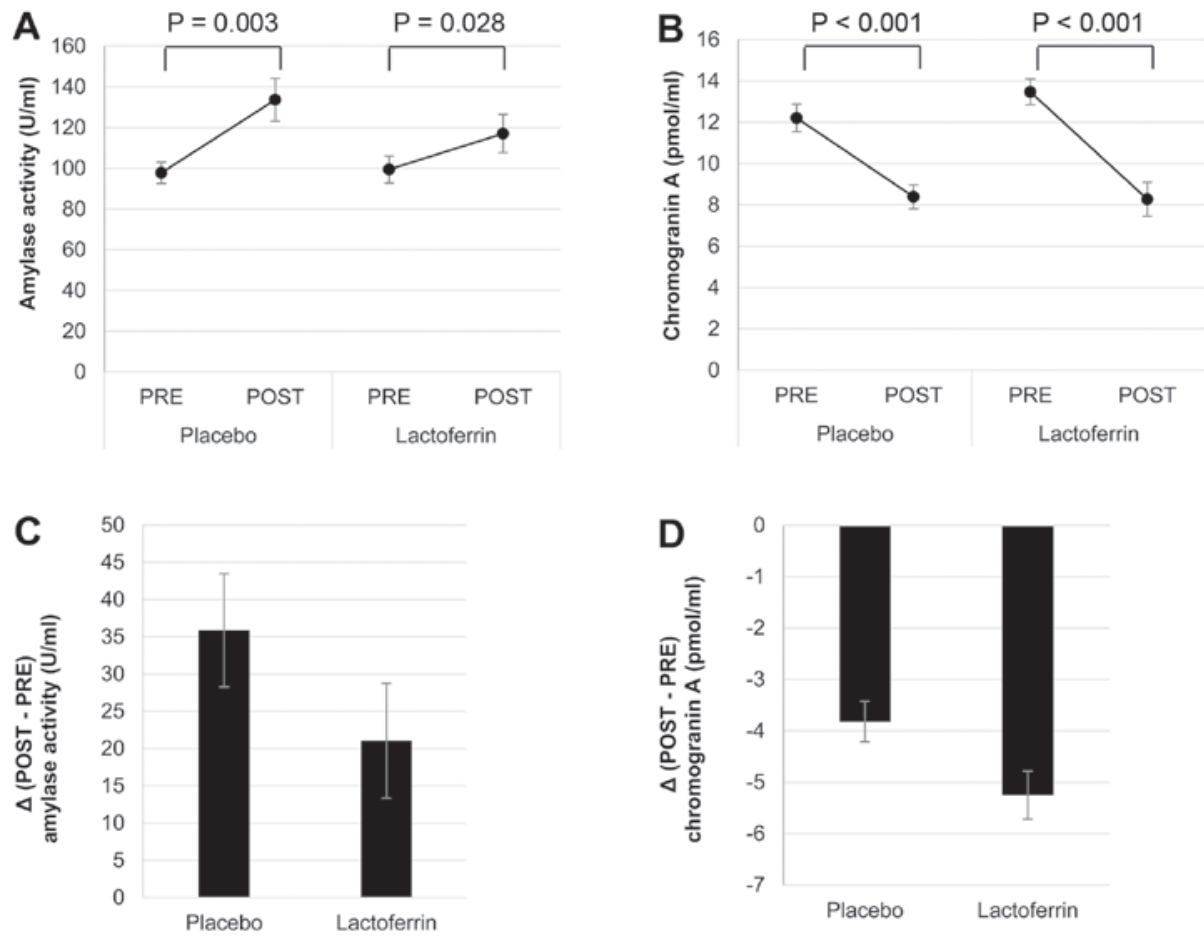

Figure 2. Changes in salivary stress markers induced by the calculation task. (A) Amylase activity. (B) Chromogranin A concentration. (C) Changes in amylase activity (POST-PRE). (D) Changes in chromogranin A concentration (POST-PRE). PRE, pre-condition; POST, post-condition.

the $\mathrm{LF} / \mathrm{HF}$ and $\mathrm{LF} /(\mathrm{LF}+\mathrm{HF})$ ratios were reduced (both $\mathrm{P}<0.001$; Fig. 3C and D). By contrast, with the lactoferrin ingestion, the LF component decreased ( $\mathrm{P}=0.034$; Fig. 3A), while the HF component remained similar (Fig. 3B) following the calculation task. Accordingly, only the $\mathrm{LF} /(\mathrm{LF}+\mathrm{HF})$ ratio decreased ( $\mathrm{P}=0.004$; Fig. 3D), without a significant change in the $\mathrm{LF} / \mathrm{HF}$ ratio (Fig. 3C).

When changes in heart-rate variability parameters were expressed as ratios (Calc 2/PRE; Fig. 4), lactoferrin ingestion was identified to significantly attenuate the elevation in the HF component $(\mathrm{P}=0.007$; Fig. $4 \mathrm{~B})$ and the reduction in the $\mathrm{LF} /(\mathrm{LF}+\mathrm{HF})$ ratio $(\mathrm{P}=0.026$; Fig. $4 \mathrm{D})$ compared with the placebo treatment.

\section{Discussion}

The current study investigated the effect of oral lactoferrin ingestion on changes in salivary stress markers and autonomic nervous activities before and after solving calculation problems. For both lactoferrin and placebo treatments, the calculation task increased salivary amylase activity and decreased chromogranin A concentration, with the magnitudes of changes in these salivary markers being similar between the two treatments. Despite this, autonomic nervous activities appeared to be influenced by the ingestion of lactoferrin. With the placebo, heart-rate variability exhibited an increase in the $\mathrm{HF}$ component and decreases in the $\mathrm{LF} / \mathrm{HF}$ and $\mathrm{LF} /(\mathrm{LF}+\mathrm{HF})$ ratios, representing an upregulation of parasympathetic activity and a downregulation of sympathetic activity. Lactoferrin ingestion, however, made the changes in the HF component and $\mathrm{LF} /(\mathrm{LF}+\mathrm{HF})$ ratio significantly smaller compared with placebo.

The present study identified a decrease in chromogranin A concentration following the calculation task, which was contradictory to what was expected based on previous studies (13-15). The observed discrepancy may be due to the difference in secretory mechanisms (Fig. 5). The release of salivary amylase is affected by noradrenergic stimuli; both the sympathetic nervous system and hypothalamic-pituitary-adrenal axis (12), as well as parasympathetic nervous 
A

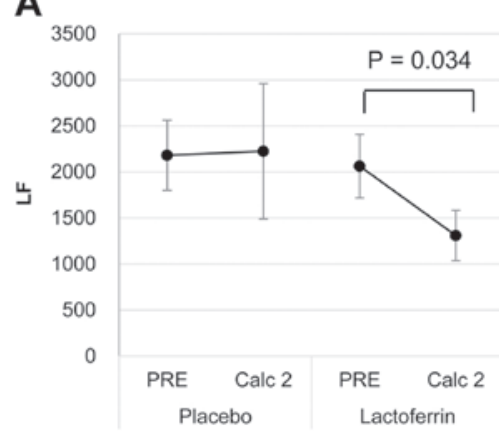

C

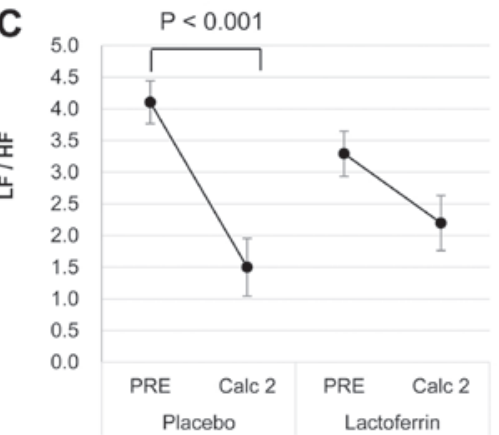

B

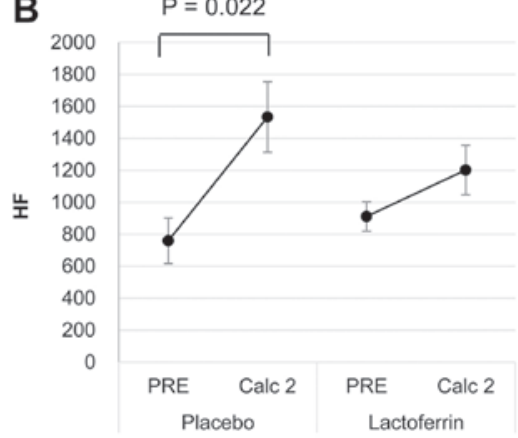

D

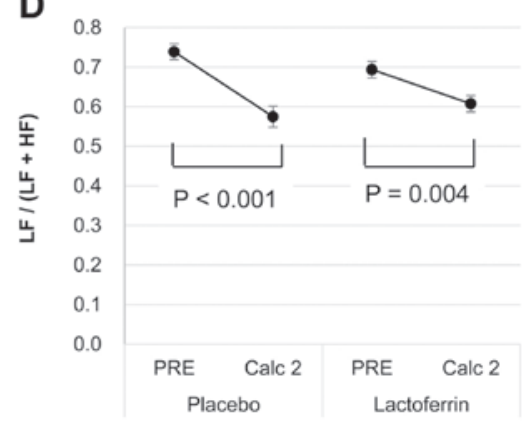

Figure 3. Changes in heart-rate variability induced by the calculation task. (A) Power of the LF component. (B) Power of the HF component. (C) LF/HF ratio. (D) $\mathrm{LF} /(\mathrm{LF}+\mathrm{HF})$ ratio. $\mathrm{LF}$, low-frequency; $\mathrm{HF}$, high-frequency.

A
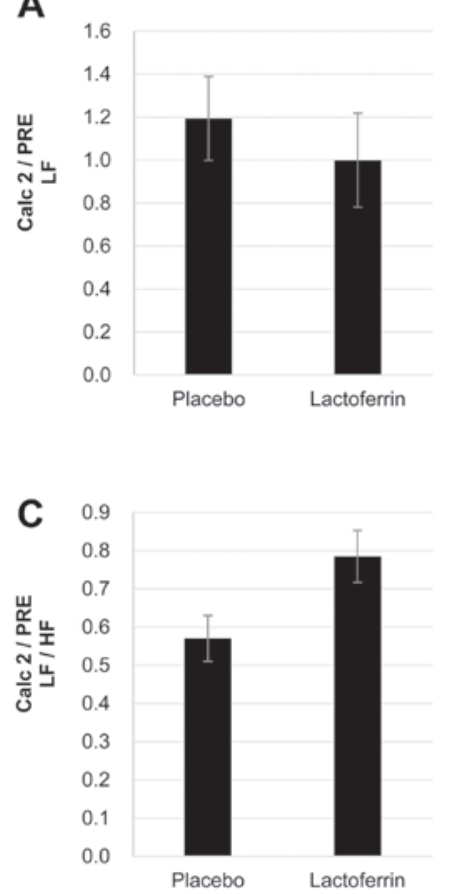

B
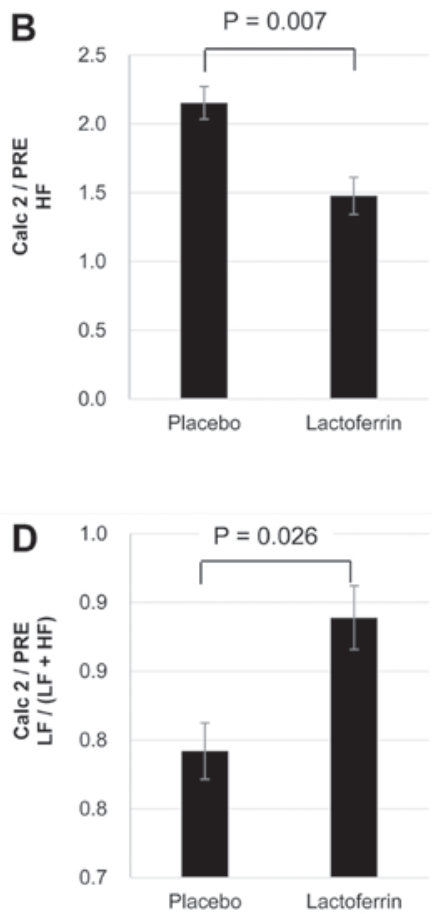

Figure 4. Changes in variability induced by the calculation task. (A) Change (Calc 2/PRE) in power of LF component. (B) Change (Calc 2/PRE) in power of HF component. (C) Change (Calc 2/PRE) in LF/HF ratio. (D) Change (Calc 2/PRE) in LF/(LF+HF) ratio. LF, low-frequency; HF, high-frequency; Calc, calculation task; PRE, pre-condition.

activity (22). An association of parasympathetic activity and amylase activity has been identified in previous study: A rise in amylase activity was observed during relaxation (23). Subsequently, exercise and psychological stress were also observed to increase amylase activity $(12,16)$ Predominantly, parasympathetic nerves stimulate amylase release from the palate, sublingual and minor palatine glands, whereas parasympathetic and sympathetic nerves collaboratively promote amylase secretion from the submandibular and parotid glands (22). By contrast, chromogranin A is stored in the 


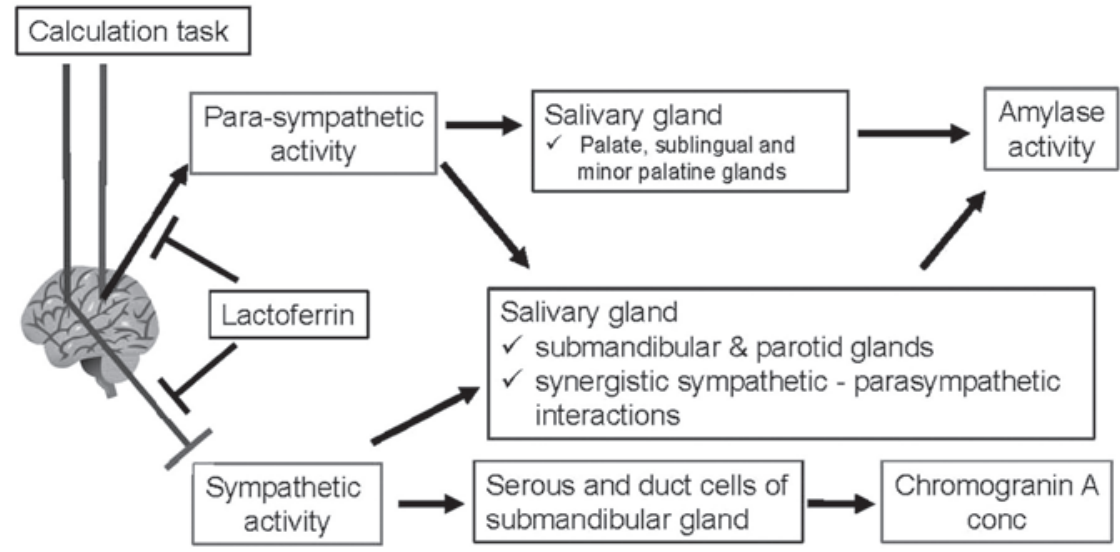

Figure 5. Mechanism of autonomic nervous activities in stimulating salivary amylase activity and chromogranin A concentration, and the effect of the calculation task and lactoferrin.

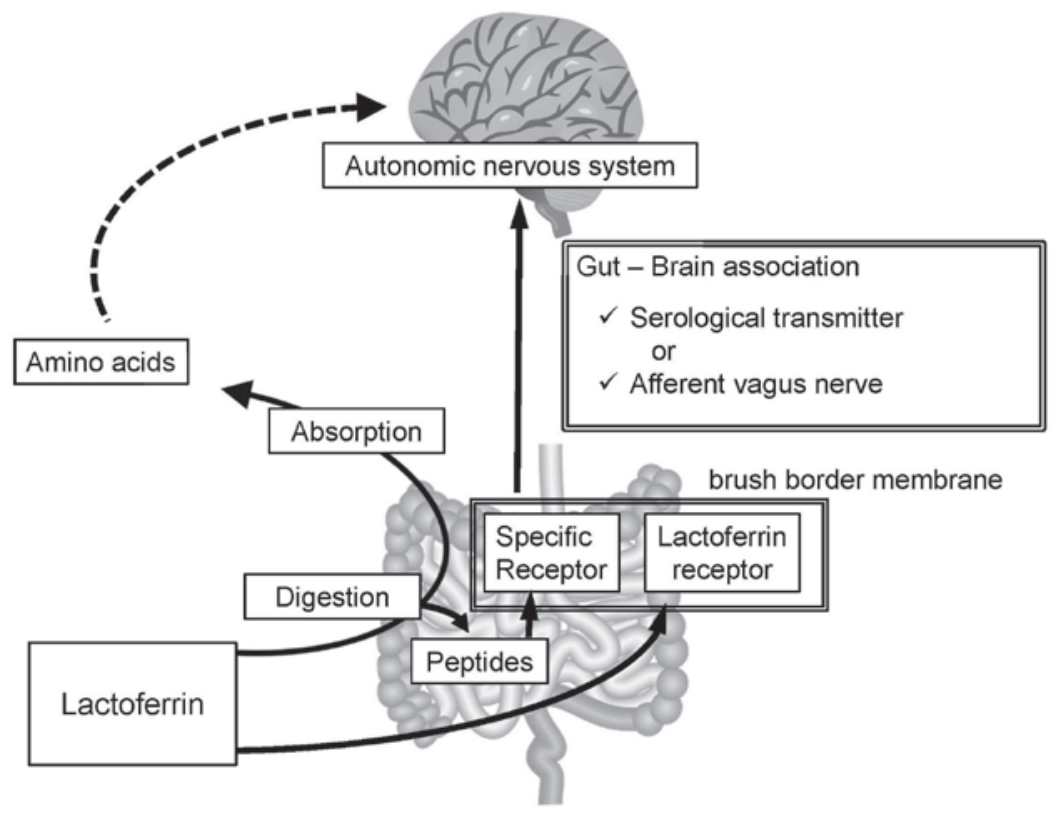

Figure 6. Hypothesized signal transduction pathway from oral lactoferrin to the autonomic nervous system.

serous and duct cells of the submandibular gland (24), and is secreted into saliva by sympathetic noradrenergic stimulation. Therefore, the secretions of amylase and chromogranin A are controlled by differing innervation patterns.

The present study demonstrated that, without lactoferrin, the calculation task raised HF (parasympathetic activity), but did not influence LF, resulting in a reduced LF/HF ratio (sympathetic activity). These results, including the change in amylase activity, were in agreement with a previous report using the TSST to induce psychological stress (18). Therefore, the calculation task was assumed to stimulate parasympathetic activity to upregulate amylase activity, and suppress sympathetic activity to downregulate chromogranin A secretion (Fig. 5). Oral lactoferrin ingestion, however, diminished LF activity and suppressed the rise in HF (parasympathetic) activity, resulting in a smaller decline in LF/HF ratio (sympathetic activity). Accordingly, this made the changes (Calc 2/PRE) in parasympathetic (HF) and sympathetic ( $\mathrm{LF} /(\mathrm{LF}+\mathrm{HF})$ ratio) activities smaller than the placebo. Therefore, oral lactoferrin ingestion may have alleviated the changes in autonomic nervous activity evoked by the calculation task (Fig. 5).

As lactoferrin is a protein with a molecular weight of $80 \mathrm{kDa}$, it should be digested into smaller peptides or amino acids prior to absorption. Certain reports have described milk-derived peptides exerting biological activities other than nutritive value (25-28). There may be a peptide derived from lactoferrin that binds to a specific receptor to modulate autonomic nervous activity; however, the absorbed amino acids are unlikely to have such specific roles (Fig. 6).

In the human intestine, lactoferrin has a specific receptor on the brush border membrane (29), namely intelectin-1, a carbohydrate-binding protein with a fibrinogen-like fold and calcium-binding site (30). Intestinal introduction of Lactobacillus rhamnosus (L. rhamnosus) has been demonstrated to augment afferent vagus activity within minutes, though this effect was abolished if subdiaphragmatic vagotomy had already been performed (31). The bacterium was considered to stimulate transmission of signal from the 
intestinal lumen to the autonomic nervous system via the afferent vagus nerve. As the lactoferrin receptor, intelectin-1, has a carbohydrate-binding activity (30), the cell wall of introduced L. rhamnosus may bind to intelectin-1. Therefore, intelectin-1 may transmit the signal from lactoferrin and L. rhamnosus, or there may be other receptors on the brush border membrane.

Intestinal cells transmit signals via serological messengers or nervous activity. Intestinal cells secrete hormones including ghrelin, cholecystokinin and glucagon-like peptide-1 in response to nutrients in the intestinal tract (32), whereas the afferent vagus nerve transmits the signal directly to the brain (31). Therefore, lactoferrin or lactoferrin-derived peptides may bind to specific receptors and modulate the autonomic nervous activity (Fig. 6).

Limitations to the current study must be considered. Firstly, lactoferrin did not exert apparent effects on salivary stress markers. A previous report demonstrated that salivary amylase activity increased when subjects viewed a stressful video, but normalized immediately following the end of viewing (33). The current study collected saliva following the second set of calculation tests. The time schedule applied may have been unsuited to observe the effects of the calculation stimuli. Salivary amylase activity and chromogranin A concentration also have distinct circadian rhythms $(34,35)$, which may have confounded the current results. Secondly, the intestinal microbiota was not assessed, despite its importance in the gut-brain association (36-38), and the fact that lactoferrin may exert effects as a bactericidal protein (1-4). Thirdly, while certain possible mechanisms by which lactoferrin may influence autonomic nervous activity have been considered, the actual mechanisms remain to be elucidated. Finally, subjects in the study were healthy female college students with homogeneous background characteristics, which potentially limits the generalizability of the conclusions.

A single-dose cross-over study was conducted to assess the influence of oral lactoferrin on psychological stresses incurred by a calculation task. The calculation task resulted in upregulated parasympathetic activity that increased salivary amylase activity, and downregulated sympathetic activity that reduced chromogranin A concentration. Oral lactoferrin ingestion suppressed the changes in parasympathetic and sympathetic activities evoked by the calculation task. These findings indicate the possible application of lactoferrin in managing psychological stress.

\section{Acknowledgements}

The images of brain and intestine in Figs. 5 and 6 were provided by DBCLS Togo Picture Gallery ( ${ }^{\odot} 2016$ DBCLS TogoTV; http://togotv.dbcls.jp/ja/pics.html, accessed on Feb 16, 2018). The authors would like to thank Dr Akihiro Sakamoto for his invaluable advice.

\section{Funding}

The present study was supported by the Cross-Ministerial Strategic Innovation Promotion Program of the Cabinet Office, Government of Japan (grant no. 14532924).

\section{Availability of data and materials}

The datasets used and/or analyzed during the current study are available from the corresponding author on reasonable request.

\section{Authors' contributions}

ST analyzed and interpreted the data and wrote the draft manuscript. TI and MK collected the data. KS and AN were involved in data collection and critically edited the manuscript. YA analyzed the salivary chromogranin A concentrations. YS designed and managed the study. All authors read and approved the final version of the manuscript.

\section{Ethics approval and consent to participate}

The research protocol was reviewed and approved by the research ethics committee of the Graduate School of Health and Sports Science, Juntendo University (Inzai, Japan; approval no. 27-54).

\section{Consent for publication}

All subjects provided written informed consent to participate in the study and permitting publication of relevant data following anonymization of personally identifiable information.

\section{Competing interests}

All authors declare that they have no competing interests.

\section{References}

1. Arnold RR, Cole MF and McGhee JR: A bactericidal effect for human lactoferrin. Science 197: 263-265, 1977.

2. Arnold RR, Brewer M and Gauthier JJ: Bactericidal activity of human lactoferrin: Sensitivity of a variety of microorganisms. Infect Immun 28: 893-898, 1980.

3. Kalmar JR and Arnold RR: Killing of Actinobacillus actinomycetemcomitans by human lactoferrin. Infect Immun 56: 2552-2557, 1988

4. Yamauchi K, Tomita M, Giehl TJ and Ellison RT III: Antibacterial activity of lactoferrin and a pepsin-derived lactoferrin peptide fragment. Infect Immun 61: 719-728, 1993.

5. Vitetta L, Coulson S, Beck SL, Gramotnev H, Du S and Lewis S: The clinical efficacy of a bovine lactoferrin/whey protein Ig-rich fraction $(\mathrm{Lf} / \mathrm{IgF})$ for the common cold: A double blind randomized study. Complement Ther Med 21: 164-171, 2013.

6. Ishikado A, Uesaki S, Suido H, Nomura Y, Sumikawa K, Maeda M, Miyauchi M, Takata T and Makino T: Human trial of liposomal lactoferrin supplementation for periodontal disease. Biol Pharm Bull 33: 1758-1762, 2010.

7. Kuhara T, Iigo M, Itoh T, Ushida Y, Sekine K, Terada N, Okamura $\mathrm{H}$ and Tsuda $\mathrm{H}$ : Orally administered lactoferrin exerts an antimetastatic effect and enhances production of IL-18 in the intestinal epithelium. Nutr Cancer 38: 192-199, 2000.

8. Koikawa N, Nagaoka I, Yamaguchi M, Hamano H, Yamauchi K and Sawaki K: Preventive effect of lactoferrin intake on anemia in female long distance runners. Biosci Biotechnol Biochem 72: 931-935, 2008 .

9. Paesano R, Torcia F, Berlutti F, Pacifici E, Ebano V, Moscarini M and Valenti P: Oral administration of lactoferrin increases hemoglobin and total serum iron in pregnant women. Biochem Cell Biol 84: 377-380, 2006.

10. Kamemori N, Takeuchi T, Hayashida K and Harada E: Suppressive effects of milk-derived lactoferrin on psychological stress in adult rats. Brain Res 1029: 34-40, 2004. 
11. Rohleder N, Wolf JM, Maldonado EF and Kirschbaum C: The psychosocial stress-induced increase in salivary alpha-amylase is independent of saliva flow rate. Psychophysiology 43: 645-652, 2006.

12. Granger DA, Kivlighan KT, el-Sheikh M, Gordis EB and Stroud LR: Salivary $\alpha$-amylase in biobehavioral research: Recent developments and applications. Ann N Y Acad Sci 1098: 122-144, 2007.

13. Haririan H, Bertl K, Laky M, Rausch WD, Böttcher M Matejka M, Andrukhov O and Rausch-Fan X: Salivary and serum chromogranin $\mathrm{A}$ and $\alpha$-amylase in periodontal health and disease. J Periodontol 83: 1314-1321, 2012.

14. Lee T, Shimizu T,Iijima M, Obinata K, Yamashiro Y and Nagasawa S Evaluation of psychosomatic stress in children by measuring salivary chromogranin A. Acta Paediatr 95: 935-939, 2006.

15. Kanno T, Asada N, Yanase H, Iwanaga T and Yanaihara N: Salivary Secretion of Chromogranin A Control by Autonomic Nervous System. In: Chromogranins: Functional and Clinical Aspects. Helle KB and Aunis D (eds). Springer US, Boston, MA pp143-151, 2002.

16. Koibuchi E and Suzuki Y: Exercise upregulates salivary amylase in humans (Review). Exp Ther Med 7: 773-777, 2014.

17. Chatterton RT Jr, Vogelsong KM, Lu YC, Ellman AB and Hudgens GA: Salivary alpha-amylase as a measure of endogenous adrenergic activity. Clin Physiol 16: 433-448, 1996.

18. Nater UM, La Marca R, Florin L, Moses A, Langhans W, Koller MM and Ehlert U: Stress-induced changes in human salivary alpha-amylase activity - associations with adrenergic activity. Psychoneuroendocrinology 31: 49-58, 2006.

19. Nakane H, Asami O, Yamada Y, Harada T, Matsui N, Kanno T and Yanaihara N: Salivary chromogranin A as an index of psychosomatic stress response. Biomed Res 19: 401-406, 1998.

20. Malik M, Bigger J, Camm A, Kleiger R, Malliani A, Moss AJ and Schwartz PJ; Task Force of the European Society of Cardiology and the North American Society of Pacing and Electrophysiology: Heart rate variability. Standards of measurement, physiological interpretation, and clinical use. Eur Heart J 17: 354-381, 1996.

21. Rajendra Acharya U, Paul Joseph K, Kannathal N, Lim CM and Suri JS: Heart rate variability: A review. Med Biol Eng Comput 44: 1031-1051, 2006.

22. Bosch JA, Veerman ECI, de Geus EJ and Proctor GB: $\alpha$-Amylase as a reliable and convenient measure of sympathetic activity: Don't start salivating just yet! Psychoneuroendocrinology 36 : 449-453, 2011.

23. Morse DR, Schacterle GR, Furst ML, Esposito JV and Zaydenburg M: Stress, relaxation and saliva: Relationship to dental caries and its prevention, with a literature review. Ann Dent 42: 47-54, 1983

24. Kanno T, Asada N, Yanase H, Iwanaga T and Yanaihara N: Salivary secretion of chromogranin A. Control by autonomic nervous system. Adv Exp Med Biol 482: 143-151, 2000.
25. Takeuchi T, Hayashida K, Inagaki H, Kuwahara M, Tsubone H and Harada E: Opioid mediated suppressive effect of milk-derived lactoferrin on distress induced by maternal separation in rat pups. Brain Res 979: 216-224, 2003.

26. Zimecki M and Kruzel ML: Milk-derived proteins and peptides of potential therapeutic and nutritive value. J Exp Ther Oncol 6: 89-106, 2007.

27. Mohanty DP, Mohapatra S, Misra S and Sahu PS: Milk derived bioactive peptides and their impact on human health - A review. Saudi J Biol Sci 23: 577-583, 2016

28. Marcone S, Belton O and Fitzgerald DJ: Milk-derived bioactive peptides and their health promoting effects: A potential role in atherosclerosis. Br J Clin Pharmacol 83: 152-162, 2017.

29. Kawakami H and Lönnerdal B: Isolation and function of a receptor for human lactoferrin in human fetal intestinal brush-border membranes. Am J Physiol 261: G841-G846, 1991.

30. Suzuki YA, Shin K and Lönnerdal B: Molecular cloning and functional expression of a human intestinal lactoferrin receptor. Biochemistry 40: 15771-15779, 2001.

31. Perez-Burgos A, Wang B, Mao YK, Mistry B, McVey Neufeld KA Bienenstock J and Kunze W: Psychoactive bacteria Lactobacillus rhamnosus (JB-1) elicits rapid frequency facilitation in vagal afferents. Am J Physiol Gastrointest Liver Physiol 304: G211-G220, 2013.

32. Steinert RE, Feinle-Bisset C, Asarian L, Horowitz M, Beglinger C and Geary N: Ghrelin, CCK, GLP-1, and PYY(3-36): Secretory Controls and Physiological Roles in Eating and Glycemia in Health, Obesity, and After RYGB. Physiol Rev 97: 411-463, 2017.

33. Takai N, Yamaguchi M, Aragaki T, Eto K, Uchihashi K and Nishikawa Y: Effect of psychological stress on the salivary cortisol and amylase levels in healthy young adults. Arch Oral Biol 49: 963-968, 2004.

34. Nater UM, Rohleder N, Schlotz W, Ehlert U and Kirschbaum C: Determinants of the diurnal course of salivary alpha-amylase. Psychoneuroendocrinology 32: 392-401, 2007.

35. Den R, Toda M, Nagasawa S, Kitamura K and Morimoto K Circadian rhythm of human salivary chromogranin A. Biomed Res 28: 57-60, 2007

36. Grenham S, Clarke G, Cryan JF and Dinan TG: Brain-gut-microbe communication in health and disease. Front Physiol 2: 94, 2011.

37. Bengmark S: Gut microbiota,immune development and function. Pharmacol Res 69: 87-113, 2013.

38. Forsythe P and Kunze WA: Voices from within: Gut microbes and the CNS. Cell Mol Life Sci 70: 55-69, 2013. 